\title{
Prognosis of proximal upper-third gastric cancer excluding tumors originating in the esophagogastric junction
}

\author{
Joong Ho Lee', Youngki Hong ${ }^{1}$, Yoon Jung Choi ${ }^{2}$, Hyunsun Lim ${ }^{3}$, Sang Hoon Lee ${ }^{1}$ \\ Departments of 'Surgery and 'Pathology, National Health Insurance Service llsan Hospital, Goyang; \\ ${ }^{3}$ Search and Analysis Team, National Health Insurance Service Ilsan Hospital, Goyang, Korea
}

Purpose: The objective of the current study was to compare surgical outcomes and prognosis based on the longitudinal location of stomach tumors in patients undergoing curative treatment. The specific focus was on the prognosis for adenocarcinomas in the proximal upper-third of the stomach, excluding tumors in the esophagogastric junction (EGJ).

Methods: Data from patients who underwent curative treatment for gastric adenocarcinoma between 2000 and 2010 at a single institution were analyzed retrospectively. Excluding tumors of EGJ origin, data from 797 patients were reviewed-686 with distal gastric cancer and 111 with proximal gastric cancer. Clinicopathology features, tumor stage, surgical outcomes, recurrence, and survival were compared between the groups.

Results: Gastric cancer recurred in 136 of the patients (17.1\%). Although differences were detected between proximal and distal cancer patients in the prevalence in males versus females $(74.8 \%$ vs. $63.4 \%, P=0.020)$ and in undifferentiated histology $(60.4 \%$ vs. $47.7 \%, P=0.013)$, the prognosis for proximal gastric cancer did not differ from distal gastric cancer. The overall 5-year disease-free survival rate was $84.9 \%$ and $81.4 \%$ in proximal and distal cancer patients, respectively $(P=0.389)$.

Conclusion: The prognosis of proximal cancer, excluding tumors of EGJ origin, did not differ from the prognosis of more distal gastric cancer. Clarifying the prognosis of proximal gastric cancer will require the large-scale comparison of an organized, multi-institution database.

Keywords: Stomach neoplasms, Recurrence, Prognosis

\section{INTRODUCTION}

The frequency of gastric cancer detected in the proximal upper-third of the stomach has increased in both the Eastern and Western hemispheres [1,2]. Existing evidence suggests that the anatomical location

Received: Aug 5, 2019 Revised: Nov 22, 2019 Accepted: Dec 5, 2019 Correspondence to: Sang Hoon Lee Department of Surgery, National Health Insurance Service Ilsan Hospital, 100 Ilsan-ro, Ilsandong-gu, Goyang 10444, Korea

Tel: +82-31-900-0216, Fax: +82-31-900-0343

E-mail: shlee618@nhimc.or.kr

ORCID: Joong Ho Lee (https://orcid.org/0000-0002-8419-1099), Youngki Hong (https://orcid.org/0000-0002-5767-9996), Yoon Jung Choi (https://orcid.org/00000002-5701-8864), Hyunsun Lim (https://orcid.org/0000-0003-2391-3286), Sang Hoon Lee (https://orcid.org/0000-0003-4819-7917)

Copyright (C) 2019 Korean Society of Surgical Oncology

This is an Open Access article distributed under the terms of the Creative Commons Attribution Non-Commercial License (http://creativecommons.org/licenses/by-nc/4.0) which permits unrestricted non-commercial use, distribution, and reproduction in any medium, provided the original work is properly cited. of stomach tumors may influence the recurrence of gastric cancer after treatment. Currently, cancer originating in the esophagogastric junction (EGI) and the cardia exhibit different lymphatic drainage than distal gastric cancer [3], leading to worse prognoses compared to tumors located in other areas of the stomach $[4,5]$.

Excepting tumors of EGJ origin, the prognosis of gastric cancer detected in the proximal upper-third of the stomach has not been established. Several studies have investigated primary tumor location and association with gastric cancer prognosis. The results of these prior studies have been contradictory, however, with some reporting a poorer prognosis in patients with a tumor in the proximal upper-third of the stomach compared with that in the distal region [6,7], whereas other studies have indicated no relationship between prognosis and the longitudinal location of the tumor in the stomach [8]. To date, no studies have reported a definitive prognosis of proximal upper-third gastric cancer excepting cancer of the EGJ.

Accordingly, the focus of the current study was on the prognosis and clinicopathological outcomes of adenocarcinoma of the prox- 
imal stomach, excluding tumors in the EGJ. Our objective was to determine recurrence patterns according to the longitudinal location of the stomach tumor. We also investigated potentially predictive independent prognostic factors of recurrence after curative treatment for gastric cancer.

\section{METHODS}

\section{Patients}

We conducted a single-center, retrospective review of 885 patients who underwent curative radical gastrectomy and adjuvant therapy for gastric cancer from March 2000 through March 2010. Patients who underwent curative surgery were included, whereas patients with distant metastases or those that underwent palliative surgery or 30-day postoperative mortalities were excluded. To meet our research objectives, patients with cancer originating in the cardia or EGJ were likewise excluded (Siewert types I and II tumors were excluded from the analysis). Finally, patients with tumors that involved the entire stomach and with no identifiable epicenter were excluded. Details of the exclusion criteria are provided in Fig. 1.

Patient demographics and clinical pathology characteristics, including tumor location, depth of tumor invasion, histological type, number of positive lymph nodes, and information regarding recurrence were obtained from a gastric cancer patient database and retrospectively reviewed at the National Health Insurance Service Ilsan Hospital, Goyang, Republic of Korea. Approval was obtained from the Hospital Institutional Review Board (NHIMC 2017-07-020).

\section{Patient categories}

The patients were divided into two groups based on the location of the tumor epicenter. The proximal cancer group contained patients

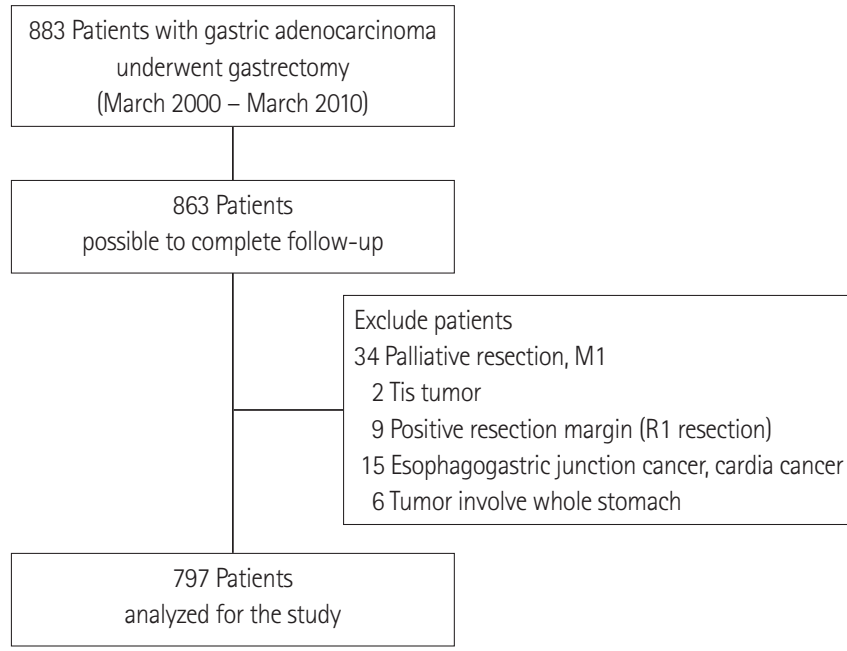

Fig. 1. The eligibility criteria of patients. Tis tumor, in situ tumor. with tumors in the proximal upper one-third of the stomach and the distal cancer group contained patients with tumors in the middle- and lower-third of the stomach. Proximal cancer was defined by the location of the tumor epicenter in the fundus or upper body of the stomach, excluding tumors in the cardia or EGJ. To accurately confirm the tumor epicenter, we reviewed the surgical pathology report for each patient. The location of the lesion was not clearly confirmed in the pathology report for some patients; thus, the preoperative examination results, including endoscopy and computed tomography $(\mathrm{CT})$, were reviewed to determine the location.

\section{Surgery and follow-up schedules}

The patients underwent gastrectomy with D1+ or D2 lymphadenectomy according to clinical stage. The indication of gastrectomy and extent of lymph node dissection was determined based on the Korean and Japanese Gastric Cancer Association general guidelines for gastric cancer treatment $[9,10]$. After curative surgery, the patients were followed every 3 months during the first 2 years and every 6 months during the following 3 years. In general, each follow-up visit included a physical examination, chest X-ray, and laboratory tests including tumor markers. Abdominopelvic CT was performed every 6 months during the first 3 years and annually at follow-up over the next 2 years. Esophagogastroduodenoscopy (EGD) was conducted annually. If a patient was lost to follow-up, we collected survival data from the National Statistical Office of Korea.

\section{Data analysis}

Analyses of clinicopathology data was performed using SAS software, version 9.4 (SAS Institute, Cary, NC, USA). The correlations between tumor recurrence and patient pathology parameters were determined using chi-square and Fisher exact tests, as appropriate. To identify the independent risk factors for recurrence after curative gastrectomy, a multivariate logistic regression model was used. Disease-free and overall survival curves were generated using the Kaplan-Meier method and compared using a log-rank test. P-values less than 0.05 were considered statistically significant.

\section{RESULTS}

\section{Demographics}

The mean age of the 518 males (65.0\%) and 279 females (35.0\%) included in this study (797 patients total) was 61.0 years. The mean follow-up period was 71.25 months. The most prevalent tumor location was the distal stomach $(\mathrm{n}=686,86.1 \%)$, and 111 of the patients (13.9\%) had proximal tumors. The majority of the patients included in the study ( $\mathrm{n}=620,77.8 \%)$ underwent distal subtotal gastrectomy.

No significant differences were detected in the mean age of the 
patients in the proximal and distal cancer groups. However, the prevalence of proximal tumors was significantly higher than that of distal tumors in males $(74.8 \%$ vs. $63.4 \%, \mathrm{P}=0.020)$. In regard to the surgical resection range of the stomach, patients with proximal cancer underwent total (91.9\%) or proximal (8.1\%) gastrectomy, whereas the majority of the patients with distal cancers underwent distal gastrectomy (90.4\%). A total gastrectomy was performed in $9.6 \%$ of the patients in the distal cancer group.

\section{Clinicopathology characteristics}

The clinicopathologic characteristics of patients and stomach tumors of proximal and distal origin are compared in Table 1. The

Table 1. Comparison of clinicopathological and surgical characteristics of distal and proximal gastric cancers

\begin{tabular}{|c|c|c|c|c|}
\hline Variable & $\begin{array}{c}\text { Total } \\
(n=797)\end{array}$ & $\begin{array}{c}\text { Distal cancer } \\
(n=686,86.1 \%)\end{array}$ & $\begin{array}{l}\text { Proximal cancer } \\
(n=111,13.9 \%)\end{array}$ & P-value \\
\hline Age (yr) & $61.0 \pm 12.0$ & $61.3 \pm 11.9$ & $59.2 \pm 12.8$ & 0.092 \\
\hline Sex & & & & 0.020 \\
\hline Male & $518(65.0)$ & $435(63.4)$ & $83(74.8)$ & \\
\hline Female & $279(35.0)$ & $251(36.6)$ & $28(25.2)$ & \\
\hline Tumor size (mm) & $41.4 \pm 27.4$ & $40.7 \pm 27.1$ & $45.6 \pm 28.7$ & 0.080 \\
\hline Retrieved lymph node & $35.9 \pm 17.7$ & $34.8 \pm 17.2$ & $42.4 \pm 19.5$ & $<0.001$ \\
\hline Depth of invasion & & & & 0.282 \\
\hline Mucosa \& submucosa (T1) & $387(48.6)$ & $343(50.0)$ & $44(39.6)$ & \\
\hline Proper muscle (T2) & $116(14.6)$ & $97(14.1)$ & 19 (17.1) & \\
\hline Subserosa (T3) & $138(17.3)$ & $113(16.5)$ & $25(22.5)$ & \\
\hline Invades serosa (T4a) & $134(16.8)$ & $115(16.8)$ & $19(17.1)$ & \\
\hline Invades adjacent structures (T4b) & $22(2.8)$ & $18(2.6)$ & $4(3.6)$ & \\
\hline Lymph node metastasis & & & & 0.402 \\
\hline Negative & $481(60.4)$ & $410(59.8)$ & $71(64.0)$ & \\
\hline Positive & $316(39.6)$ & $276(40.2)$ & $40(36.0)$ & \\
\hline Pathological stage, AJCC 8th edition & & & & 0.147 \\
\hline । & $433(54.3)$ & $378(55.1)$ & 55 (49.5) & \\
\hline$\|$ & $154(19.3)$ & $125(18.2)$ & $29(26.1)$ & \\
\hline III & $210(26.3)$ & $183(26.7)$ & $27(24.3)$ & \\
\hline Differentiation & & & & 0.013 \\
\hline Differentiated & $403(50.6)$ & $359(52.3)$ & $44(39.6)$ & \\
\hline Undifferentiated & $394(49.4)$ & $327(47.7)$ & $67(60.4)$ & \\
\hline Lauren classification & & & & 0.192 \\
\hline Intestinal & $304(38.1)$ & 269 (39.2) & $35(31.5)$ & \\
\hline Diffuse & $470(59.0)$ & $396(57.7)$ & $74(66.7)$ & \\
\hline Mixed & $23(2.9)$ & $21(3.1)$ & $2(1.8)$ & \\
\hline Lymphovascular invasion & & & & 0.602 \\
\hline None & $492(61.7)$ & $421(61.4)$ & $71(64.0)$ & \\
\hline Present & $305(38.3)$ & $265(38.6)$ & $40(36.0)$ & \\
\hline Perineural invasion & & & & 0.788 \\
\hline None & $646(81.1)$ & $555(80.9)$ & $91(82.0)$ & \\
\hline Present & $151(18.9)$ & $131(19.1)$ & $20(18.0)$ & \\
\hline Extent of gastrectomy & & & & $<0.001$ \\
\hline Distal gastrectomy & $620(77.8)$ & $620(90.4)$ & 0 & \\
\hline Total gastrectomy & $168(21.1)$ & $66(9.6)$ & $102(91.9)$ & \\
\hline Proximal gastrectomy & $9(1.1)$ & 0 & $9(8.1)$ & \\
\hline Adjuvant chemotherapy & & & & 0.658 \\
\hline None & $483(60.6)$ & $420(61.2)$ & $63(56.8)$ & \\
\hline Single regimen & $226(28.4)$ & $192(28.0)$ & $34(30.6)$ & \\
\hline Combination regimen & 88 (11.0) & 74 (10.8) & $14(12.6)$ & \\
\hline Recurrence & $136(17.1)$ & $120(17.5)$ & $16(14.4)$ & 0.424 \\
\hline
\end{tabular}

Values are presented as mean \pm standard deviation or number $(\%)$.

AJCC, American Joint Committee on Cancer. 
total number of retrieved lymph nodes in the proximal cancer group were higher than that of the distal cancer group $(42.4 \pm 19.5$ vs. $34.8 \pm 17.2, \mathrm{P}<0.001)$. Also, the frequency of undifferentiated tumors was relatively higher in the proximal cancer group than in the distal cancer group ( $60.4 \%$ vs. $47.7 \%, \mathrm{P}=0.013)$. Although the differences were not statistically significant, tumor sizes in the proximal cancer group were larger than those in the distal cancer group (45.6 mm vs. $40.7 \mathrm{~mm}, \mathrm{P}=0.080$ ) Additionally, no significant differences in the distribution of lymphovascular or perineural invasion, the number of lymph node metastases, or the pathologic stage were detected between the distal and proximal cancer groups $(\mathrm{P}>0.05)$.

\section{Recurrence}

No difference in recurrence rate was detected between the proximal and distal cancer groups ( $14.4 \%$ vs. $17.5 \%$, respectively, $\mathrm{P}=0.424)$. One hundred and thirty-six patients (17.1\%) experienced recurrence during the follow-up periods. The mean length of time to recurrence was 22.6 months. Of these patients, 88 (64.7\%) experienced recurrence at 2 years and 131 (96.3\%) experienced recurrence at 5 years after curative surgery. Five patients (3.7\%) were diagnosed with recurrence after 5 years, post-surgery, and the mean months to the time of recurrence was 81.6 months (range, 65-111 months). The time of recurrence between the proximal and distal cancer groups was not statistically different $(\mathrm{P}=0.696)$.

\section{Analysis of recurrence risk factors}

The investigation of the risk factors for recurrence is shown in Table 2. The results of a univariate analysis indicated that tumor size, histological type, presence of lymphovascular or perineural inva-

Table 2. Univariate and multivariate analysis of clinicopathologic factors associated with 5-year disease-free survival

\begin{tabular}{|c|c|c|c|c|c|}
\hline \multirow{2}{*}{ Factor } & \multirow{2}{*}{ No. (\%) } & \multicolumn{2}{|c|}{ Univariable } & \multicolumn{2}{|c|}{ Multivariable } \\
\hline & & $\mathrm{HR}(95 \% \mathrm{Cl})$ & P-value & HR $(95 \% \mathrm{Cl})$ & P-value \\
\hline \multicolumn{6}{|l|}{ Age (yr) } \\
\hline$<60$ & 305 (38.3) & Reference & & & \\
\hline$\geq 60$ & $492(61.7)$ & $1.246(0.871-1.784)$ & 0.229 & & NA \\
\hline \multicolumn{6}{|l|}{ Sex } \\
\hline Male & $518(65.0)$ & Reference & & & \\
\hline Female & $279(35.0)$ & $1.106(0.776-1.576)$ & 0.577 & & NA \\
\hline \multicolumn{6}{|l|}{ Tumor location } \\
\hline Distal cancer & $686(86.1)$ & Reference & & & \\
\hline Proximal cancer & 111 (13.9) & $0.789(0.461-1.351)$ & 0.387 & & NA \\
\hline \multicolumn{6}{|l|}{ Extent of gastrectomy } \\
\hline Distal gastrectomy & $620(77.8)$ & Reference & & & \\
\hline Total \& Proximal gastrectomy & $177(22.2)$ & $2.114(1.474-3.032)$ & $<0.001$ & 1.421 (0.983-2.054) & 0.062 \\
\hline \multicolumn{6}{|l|}{ Tumor size $(\mathrm{mm})$} \\
\hline$<30$ & $288(36.1)$ & Reference & & & \\
\hline$\geq 30$ & $509(63.9)$ & 6.395 (3.532-11.580) & $<0.001$ & $1.272(0.660-2.452)$ & 0.473 \\
\hline \multicolumn{6}{|l|}{ Histology } \\
\hline Differentiated & $403(50.6)$ & Reference & & & \\
\hline Undifferentiated & $394(49.4)$ & 2.051 (1.433-2.936) & $<0.001$ & $1.182(0.812-1.721)$ & 0.382 \\
\hline \multicolumn{6}{|l|}{ Lymphovascular invasion } \\
\hline None & $492(61.7)$ & Reference & & & \\
\hline Present & $305(38.3)$ & $6.834(4.544-10.277)$ & $<0.001$ & 1.615 (0.999-2.611) & 0.05 \\
\hline \multicolumn{6}{|l|}{ Perineural invasion } \\
\hline None & 646 (81.1) & Reference & & & \\
\hline Present & 151 (18.9) & 4.351 (3.080-6.147) & $<0.001$ & 1.119 (0.769-1.628) & 0.556 \\
\hline \multicolumn{6}{|l|}{ Depth of invasion } \\
\hline $\mathrm{T} 1 \& \mathrm{~T} 2$ & $503(63.1)$ & Reference & & & \\
\hline $\mathrm{T} 3$ \& T4 & $294(36.9)$ & 15.244 (9.153-25.389) & $<0.001$ & 6.091 (3.404-10.898) & $<0.001$ \\
\hline \multicolumn{6}{|l|}{ Lymph node metastasis } \\
\hline None & $481(60.4)$ & Reference & & & \\
\hline Present & $316(39.6)$ & 10.305 (6.458-16.442) & $<0.001$ & 3.175 (1.833-5.501) & $<0.001$ \\
\hline
\end{tabular}

$\mathrm{HR}$, hazard ratio; $\mathrm{Cl}$, confidence interval; $\mathrm{NA}$, not available. 

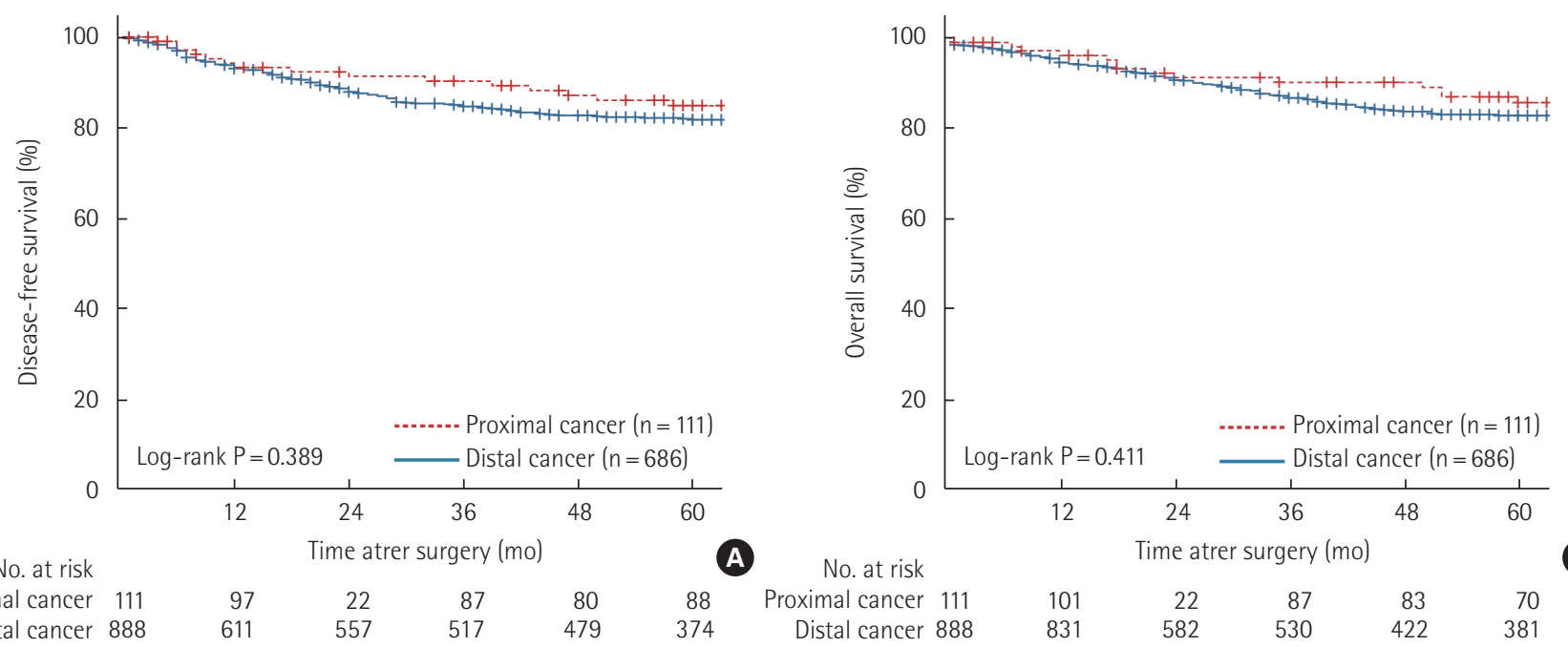

Fig. 2. Cumulative disease-free survival (A) and overall survival (B) curves of 797 patients with gastric cancer according to the longitudinal location of the tumor.

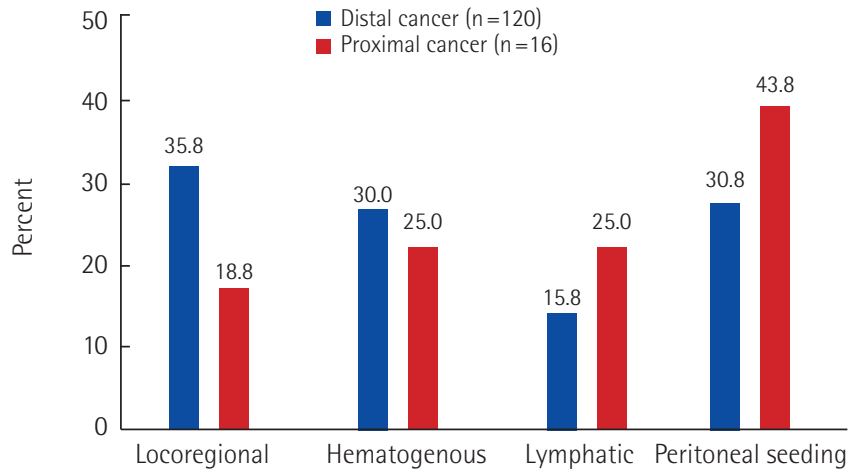

Fig. 3. The comparison of recurrence site patterns in 136 patients following a curative treatment for gastric cancer based on the longitudinal location of the tumor. The statistical values were not significantly different within each group $(P>0.05)$.

sion, extent of gastrectomy, depth of primary tumor invasion, and lymph node metastasis were risk factors for recurrence of gastric cancer, whereas age, sex, and longitudinal tumor location did not affect recurrence. Using multivariate logistic regression, we demonstrated that only the depth of invasion (hazard ratio [HR], 6.091; 95\% confidence interval [CI], 3.404-10.898; $\mathrm{P}<0.001$ ) and lymph node metastasis (HR, 3.175; 95\% CI, 1.833-5.501; $\mathrm{P}<0.001$ ) were independent risk factors for overall recurrence.

\section{Cumulative incidence of recurrence and death}

The cumulative disease-free and overall survival of patients according to tumor location are illustrated in Fig. 2. The 5-year disease-free survival rate was $81.9 \%$ (proximal vs. distal cancer: $84.9 \%$ vs. $81.4 \%$, respectively, $\log$-rank $\mathrm{P}=0.389$ ) and the disease-specific 5 -year overall survival rate was $82.9 \%$ (proximal vs. distal cancer:
$84.3 \%$ vs. $82.8 \%$, respectively, $\log$-rank $\mathrm{P}=0.411$ ). Locoregional relapse was most common (33.8\%), followed by peritoneal metastases (32.4\%), hematogenous distant metastasis (29.4\%), and lymphatic metastasis (16.9\%). The recurrence sites in patients $(\mathrm{n}=136)$ based on the longitudinal location of the tumors is shown in Fig. 3. No statistically significant differences in the site of recurrence according to tumor location were observed $(\mathrm{P}>0.05)$. However, the frequency of locoregional and hematogenous recurrence was higher in the distal cancer group; on the contrary, recurrence involving the lymphatics and peritoneal seeding was more frequent in the proximal cancer group.

\section{DISCUSSION}

This study describes recurrence patterns according to the longitudinal location of the tumors and the adverse effects of clinicopathological factors on prognosis following curative gastrectomy for gastric cancer patients at a single institution. In this study, although differences in clinicopathology were detected between proximal gastric cancer excluding tumors of EGJ origin and more distal gastric cancer, including prevalence in male gender and undifferentiated histology, there were no significant differences in the distribution of tumor size, or TNM stage, which is similar to a previously published report [7,11-13]. However, the survival rate and prognosis of proximal gastric cancer was not different from distal gastric cancer in the current study.

The depth of tumor invasion and the degree of lymph node metastasis were the most important prognostic factors of recurrence after curative treatment for gastric cancer in the present study. However, the longitudinal location of the stomach tumor did not 
affect gastric cancer recurrence. Previously, several studies have reported on the prognosis of patients with a tumor in the proximal upper-third of the stomach compared with that in the distal region [8,11-15]. Petrelli et al. [14] review of 50 studies encompassing 128,268 patients with gastric cancer and tumors in the proximal upper-third of the stomach reported an increased risk of tumor-related death, suggesting that the longitudinal location of the tumor was an important stratification prognostic factor. Also, Yu et al. [11] report on 964 patients that underwent gastrectomy for gastric cancer indicated that patients with proximal cancer exhibited the characteristics of late-stage disease, underwent more complex surgical procedures, and had worse prognoses, whereas patients with distal cancers were more likely to be diagnosed at a younger age and receive better prognoses.

The results of this study, however, indicate that despite differences in undifferentiated tumors and a prevalence in males, the prognosis of proximal gastric cancer is not different compared with distal gastric cancer. The 5 -year disease-survival rates were $84.9 \%$ for proximal gastric cancer patients and $81.4 \%$ for distal gastric cancer patients $(\mathrm{P}=0.389)$. Similarly, no differences were found in the 5 -year overall survival rate between proximal and distal gastric cancer $(84.3 \%$ vs. $82.8 \%, \mathrm{P}=0.411)$.

One reason why the results of this study differed from previous studies is that tumors of the cardia or EGJ origin were excluded from the analysis to confirm the prognosis of actual proximal body cancer. Excluding tumors of EGJ origin resulted in a slight improvement in the 5-year disease-free and overall survival rates of patients with proximal gastric cancer compared with patients with more distal cancer. A likely explanation for the improved prognosis of proximal cancer over distal cancer is the consequent broader lymph node dissection range due to a wider resection range in the stomach.

It is accepted that the prognosis for tumors of EGJ origin is poor compared with tumors located in the distal stomach $[4,5]$; thus, EGJ tumors are reported as a category that is distinct from carcinoma of the rest of the stomach [16]. Cancer of the cardia and EGJ are more frequently associated with deeper gastric wall infiltration, lymph node involvement, and lymphatic vessel invasion [13]. Accordingly, the previously reported poor proximal gastric cancer prognosis could be due to the inclusion of cancer of EGJ origin.

Another reason the results of the current study differed from prior reports is that this study categorized proximal and distal gastric cancer based on the location of the tumor epicenter. In previous studies, the criteria for dividing the two groups were not clearly described and the proximal gastric cancer group may have contained large tumors in the mid-body stomach. If the patients are divided according to the extent of gastrectomy rather than based on the location of the tumor epicenter, it is difficult to obtain accurate results that are consistent with the study purpose.

In this study, total gastrectomy was performed in $9.6 \%$ of the patients classified as having mid- to distal gastric cancer due to tumor size. The results of multivariate logistic regression did not reveal any significant impact of the extent of gastrectomy on recurrence (distal gastrectomy vs. total and proximal gastrectomy: HR, 1.421; $95 \%$ CI, 0.983-2.054; $\mathrm{P}=0.062$ ). These findings differ somewhat from that of a previous study with poor prognosis in patients with proximal cancer compared with distal cancer. The reason for these results was due to excluding tumors originating in the EGJ of the proximal cancer group. We reviewed the surgical pathology report of each patient and attempted to accurately divide the patients based on the location of the tumor epicenter while excluding tumors originating in the EGJ. Thus, we could accurately compare the clinicopathological data of proximal gastric cancer with that of distal cancers.

However, there were some inherent weaknesses in the current study that should be addressed. As a retrospective, observational study conducted at a single institution, we were limited in our ability to draw definitive conclusions of clear causality of the prognostic factors according to the tumor location. Furthermore, the comparison of the patient groups by depth of tumor invasion, number of lymph node metastases, and tumor size were essential to generating clear results, but our sample size may have been too small and our study insufficiently powered to detect causality. Hence, clarifying the prognosis of proximal gastric cancer will require a largescale comparison of an organized, multi-institution database.

The survival rates of gastric cancer patients have recently increased, motivating a growing interest in the postoperative quality of life in patients with gastric cancer. The proportion of cancerous tumors in the proximal upper-third of the stomach has steadily increased, prompting the execution of surgical options that preserve function, such as proximal gastrectomy and limited lymph node dissection in patients with gastric cancer in the proximal stomach $[17,18]$. According to the results of this study, the development of a suitable physiological and minimally invasive procedure to improve the quality of life of patients that differs from the currently established surgical range of resection for proximal gastric cancer is necessary.

In conclusion, the results of this study revealed that the prognosis of proximal cancer not of EGJ origin did not differ from the prognosis of more distal gastric cancer. Although some clinicopathologic parameters including prevalence in males and undifferentiated tumors were associated with proximal upper-third gastric cancers, the longitudinal location of stomach tumors had no adverse prognostic impact following the established treatment for 
gastric cancer. Nonetheless, to further clarify the prognosis of proximal gastric cancer, a large-scale comparison of patient data from an organized, multi-institution database is required.

\section{CONFLICT OF INTEREST}

No potential conflict of interest relevant to this article was reported.

\section{ACKNOWLEDGMENTS}

This study was supported by National Health Insurance Service Ilsan Hospital grant (NHIMC2017CR064).

\section{REFERENCES}

1. Colquhoun A, Arnold M, Ferlay J, Goodman KJ, Forman D, Soerjomataram I. Global patterns of cardia and non-cardia gastric cancer incidence in 2012. Gut 2015;64:1881-8.

2. Information Committee of Korean Gastric Cancer Association. Korean gastric cancer association nationwide survey on gastric cancer in 2014. J Gastric Cancer 2016;16:131-40.

3. Rüdiger Siewert J, Feith M, Werner M, Stein HJ. Adenocarcinoma of the esophagogastric junction: results of surgical therapy based on anatomical/topographic classification in 1,002 consecutive patients. Ann Surg 2000;232:353-61.

4. Kajiyama Y, Tsurumaru M, Udagawa H, Tsutsumi K, Kinoshita Y, Ueno $\mathrm{M}$, et al. Prognostic factors in adenocarcinoma of the gastric cardia: pathologic stage analysis and multivariate regression analysis. J Clin Oncol 1997;15:2015-21.

5. Deans C, Yeo MS, Soe MY, Shabbir A, Ti TK, So JB. Cancer of the gastric cardia is rising in incidence in an Asian population and is associated with adverse outcome. World J Surg 2011;35:617-24.

6. Han DS, Suh YS, Kong SH, Lee HJ, Choi Y, Aikou S, et al. Nomogram predicting long-term survival after $\mathrm{d} 2$ gastrectomy for gastric cancer. J Clin Oncol 2012;30:3834-40.

7. Kunisaki C, Shimada H, Nomura M, Matsuda G, Otsuka Y, Ono H, et al. Surgical outcome in patients with gastric adenocarcinoma in the upper third of the stomach. Surgery 2005;137:165-71.

8. Costa LB, Toneto MG, Moreira LF. Do proximal and distal gastric tumours behave differently? Arq Bras Cir Dig 2016;29:232-5.

9. Lee JH, Kim JG, Jung HK, Kim JH, Jeong WK, Jeon TJ, et al. Clinical practice guidelines for gastric cancer in Korea: an evidence-based approach. J Gastric Cancer 2014;14:87-104.

10. Japanese Gastric Cancer Association. Japanese gastric cancer treatment guidelines 2010 (ver. 3). Gastric Cancer 2011;14:113-23.

11. Yu X, Hu F, Li C, Yao Q, Zhang H, Xue Y. Clinicopathologic characteristics and prognosis of proximal and distal gastric cancer. Onco Targets Ther 2018;11:1037-44.

12. Sakaguchi T, Watanabe A, Sawada H, Yamada Y, Tatsumi M, Fujimoto $\mathrm{H}$, et al. Characteristics and clinical outcome of proximal-third gastric cancer. J Am Coll Surg 1998;187:352-7.

13. Pacelli F, Papa V, Caprino P, Sgadari A, Bossola M, Doglietto GB. Proximal compared with distal gastric cancer: multivariate analysis of prognostic factors. Am Surg 2001;67:697-703.

14. Petrelli F, Ghidini M, Barni S, Steccanella F, Sgroi G, Passalacqua R, et al. Prognostic role of primary tumor location in non-metastatic gastric cancer: a systematic review and meta-analysis of 50 studies. Ann Surg Oncol 2017;24:2655-68.

15. Ichikawa D, Komatsu S, Kosuga T, Konishi H, Okamoto K, Shiozaki A, et al. Clinicopathological characteristics of clinical early gastric cancer in the upper-third stomach. World J Gastroenterol 2015;21:12851-6.

16. Rice TW, Gress DM, Patil DT, Hofstetter WL, Kelsen DP, Blackstone EH. Cancer of the esophagus and esophagogastric junction-major changes in the American Joint Committee on Cancer eighth edition cancer staging manual. CA Cancer J Clin 2017;67: 304-17.

17. Jung DH, Ahn SH, Park DJ, Kim HH. Proximal gastrectomy for gastric cancer. J Gastric Cancer 2015;15:77-86.

18. Kataoka K, Katai H, Mizusawa J, Katayama H, Nakamura K, Morita $\mathrm{S}$, et al. Non-randomized confirmatory trial of laparoscopy-assisted total gastrectomy and proximal gastrectomy with nodal dissection for clinical stage I gastric cancer: Japan Clinical Oncology Group Study JCOG1401. J Gastric Cancer 2016;16:93-7. 